d

EDITOR'S

CHOICE

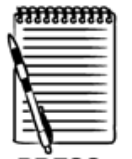

PRESS

RELEASE

\title{
Exposure to occupational noise and cardiovascular disease in the United States: the National Health and Nutrition Examination Survey 1999-2004
}

\author{
Wen Qi Gan, ${ }^{1}$ Hugh W Davies, ${ }^{1}$ Paul A Demers ${ }^{1,2}$
}

- An additional appendix is published online only. To view this file, please visit the journal online (http://oem.bmj.com/)

${ }^{1}$ School of Environmental Health, The University of British Columbia, Vancouver, Canada

${ }^{2}$ School of Population and Public Health, The University of British Columbia, Vancouver, Canada

\section{Correspondence to}

Dr Wenqi Gan, School of Environmental Health, University of British Columbia, 316 - 2206 East Mall, Vancouver, BC V6T 1Z3, Canada;

wenqi.gan@gmail.com

Accepted 12 May 2010 Published Online First

5 October 2010

\begin{abstract}
Background Chronic exposure to occupational noise may be associated with increased risk of coronary heart disease (CHD) and hypertension. However, findings are inconsistent and many previous studies are limited by small sample size and inappropriate control for potential confounders. We used a nationally representative US sample to examine associations of self-reported exposure to occupational noise with CHD and hypertension.

Methods This cross-sectional study included 6307 participants of the National Health and Nutrition Examination Survey 1999-2004, aged $\geq 20$ years and employed at the time of interview. Noise exposure assessment was based on self-reported exposure to loud noise in the workplace.
\end{abstract}

Results Compared with never exposed participants, subjects chronically exposed to occupational noise had a 2-3-fold increased prevalence of angina pectoris, myocardial infarction, $\mathrm{CHD}$ and isolated diastolic hypertension. After adjustment for various covariates, the odds ratios (95\% Cls) for angina pectoris, CHD and isolated diastolic hypertension were 2.91 (1.35 to 6.26), 2.04 (1.16 to 3.58 ) and 2.23 (1.21 to 4.12), respectively. There were clear exposure-response relationships for the observed associations. Associations of noise exposure with angina pectoris, myocardial infarction and $\mathrm{CHD}$ were particularly strong for participants aged $<50$ years, men and current smokers. There was no significant increase in levels of cardiovascular biomarkers including blood lipids and circulating inflammatory mediators associated with noise exposure.

Conclusions Chronic exposure to occupational noise is strongly associated with prevalence of CHD, especially for young male current smokers. This study suggests that excess noise exposure in the workplace is an important occupational health issue and deserves special attention.

\section{INTRODUCTION}

Excess noise is a common occupational hazard worldwide. ${ }^{1}$ In the United States, approximately 22.4 million (17.2\%) workers are exposed to hazardous occupational noise. ${ }^{2}$ It has been well documented that noise exposure can cause hearing impairment, sleep disturbance, annoyance and psychological stress. ${ }^{1}{ }^{3}$ Importantly, recent research has indicated that chronic exposure to noise is associated with an increased risk of cardiovascular diseases such as coronary heart disease (CHD) and hypertension, especially in occupational settings. ${ }^{3-6}$ However, the findings

\section{What this paper adds}

- Findings from previous studies on the associations between exposure to occupational noise and coronary heart disease are not consistent and many previous studies are limited by small sample size and inappropriate control for potential confounders.

- Self-reported chronic exposure to occupational noise was associated with increased prevalence of coronary heart disease; the association was particularly strong for participants aged less than 50 years, men and current smokers.

- Excess noise exposure in the workplace is an important occupational health issue and deserves special attention.

(eg, the association with $\mathrm{CHD}$ ) from previous studies are not consistent, ${ }^{4} 67$ and many previous studies are limited by small sample size and inappropriate control for potential confounders. ${ }^{4} 6$ In addition, previous studies of occupational noise exposure focused only on a specific industry, and their findings are difficult to generalise since the nature of the noise and actual human exposure may vary substantially across different industries. ${ }^{1}$ Given the limitations and inconsistent findings from previous studies, the relationships between exposure to occupational noise and cardiovascular outcomes remain uncertain and deserve further investigation.

We conducted a cross-sectional study using a nationally representative sample from the National Health and Nutrition Examination Survey (NHANES) 1999-2004 to examine the associations between exposure to occupational noise and the prevalence of cardiovascular diseases, especially $\mathrm{CHD}$ and hypertension. We also examined the associations between noise exposure and cardiovascular biomarkers including blood lipids and circulating inflammatory mediators.

\section{METHODS}

NHANES was a series of cross-sectional investigations of the non-institutionalised civilian population to assess the health and nutritional status of adults and children in the United States. Participants were chosen through a stratified multistage probability sampling procedure to serve as a nationally representative sample. Subgroups of the population such as the elderly, non-Hispanic 
black Americans and Mexican Americans were oversampled to provide reliable estimates for these groups. The survey included a household interview and a subsequent medical examination at a mobile examination centre, which also included laboratory tests on blood and urine. During the household interview, various health-related questionnaires including an occupation questionnaire were administered by a trained interviewer and the data were recorded on a portable laptop computer. A detailed description of the study design and sampling methodology was provided elsewhere. ${ }^{8}$ In accordance with the National Center for Health Statistics (NCHS) analytical guidelines, we aggregated the relevant data from the three consecutive surveys (NHANES 1999-2000, 2001-2002 and 2003-2004) that include occupation investigation to create a combined NHANES 1999-2004 dataset. ${ }^{9}$ For participants aged 20 years and older, the overall response rate was $75.8 \%$ for the household interview and $70.3 \%$ for the medical examination. ${ }^{10}$ The 1999-2004 NHANES was reviewed and approved by the NCHS Institutional Review Board. Informed consent was obtained from all participants.

\section{Study sample}

The present analysis was restricted to participants who were 20 years and older and were currently employed (within the previous week) at the time of interview. In the combined NHANES 1999-2004 dataset, there were 15332 participants aged 20 years and older, of whom 8152 (53.2\%) were currently employed. Of these participants, 1307 who were once exposed to loud occupational noise for at least 3 months but were currently not exposed (former exposed group) were excluded from this study due to lack of information about the duration of the exposure. We further excluded 538 participants with missing data on noise exposure time $(n=10)$, educational attainment $(n=6)$, leisure time physical activity $(n=2)$, cigarette smoking status $(n=6)$ and body mass index (BMI) $(n=518)$, leaving 6307 participants for the main analysis (model 1 and model 2).

For these participants, there was a substantial amount of missing data on annual family income $(n=501)$, pack-years of cigarette smoking $(n=370)$, passive smoking $(n=73)$, alcohol drinking $(n=469)$, waist circumference $(n=107)$ and total cholesterol level ( $n=351)$. To control for the influence of these relevant factors, we further excluded 1478 participants with missing data on these items, leaving 4829 participants for additional adjustment analysis (model 3 ).

\section{Noise exposure assessment}

According to previous and current noise exposure status in the workplace, participants were divided into two groups:

Never exposed group: defined by answering 'no' to the question: 'Thinking of all the jobs you have ever had, have you ever been exposed to loud noise at work for at least three months? By loud noise I mean noise was so loud that you had to speak in a raised voice to be heard'.

Current exposed group: participants had been exposed to loud occupational noise for at least three months and were also currently exposed at the time of interview.

For the current exposed group, accumulated exposure time was estimated using average daily exposure hours ('On average, how many hours per day are you currently exposed to this loud noise?'; if less than $1 \mathrm{~h}$, a value of 1 was entered) and the number of months working in the current job ('How long have you worked for the employer?'; if less than 1 month, a value of 0 was entered). Accumulated noise exposure time was then calculated as average hours/day $\times$ (months in the job $\times 21.67$ ) days (21.67 represents the average number of working days per month). Based on accumulated noise exposure time, participants in the current exposed group were divided into tertiles, representing short- ( $0-0.3$ years), medium- (0.4-1.5 years) and long-term (1.6-18.8 years) exposure to occupational noise, respectively. We also performed similar exposure assessment using the longest job ('Thinking of all the paid jobs or businesses you ever had, what kind of work were you doing the longest?') for which noise exposure time was available.

\section{Cardiovascular diseases and biomarkers}

Cardiovascular disease was defined as self-report of diagnosis by a doctor or other health professional unless otherwise specified (eg, self-reported CHD was defined by answering 'yes' to the question: 'Has a doctor or other health professional ever told you that you had coronary heart disease?'). CHD in this study included self-reported $\mathrm{CHD}$, angina pectoris and myocardial infarction (MI). Cardiovascular disease included CHD as well as self-reported hypertension, stroke and congestive heart failure.

In the NHANES Survey, blood pressure was measured three or four times manually using a mercury sphygmomanometer. ${ }^{11}$ Average systolic (SBP) and diastolic blood pressure (DBP) were calculated after excluding the first measurement. High blood pressure was divided into the following subtypes ${ }^{12}$ : (1) isolated systolic hypertension (ISH): SBP $\geq 140$ and DBP <90 mm Hg; (2) isolated diastolic hypertension (IDH): SBP $<140$ and DBP $\geq 90$ $\mathrm{mm} \mathrm{Hg}$; and (3) systodiastolic hypertension (SDH): SBP $\geq 140$ and $\mathrm{DBP} \geq 90 \mathrm{~mm} \mathrm{Hg}$. General hypertension was defined as SBP $\geq 140 \mathrm{~mm} \mathrm{Hg}, \mathrm{DBP} \geq 90 \mathrm{~mm} \mathrm{Hg}$, or self-reported hypertension.

Blood samples were drawn during the medical examination. Laboratory blood tests for this study included blood lipids (total cholesterol, HDL-cholesterol, LDL-cholesterol and triglyceride), circulating inflammatory mediators (C-reactive protein, fibrinogen, leukocytes and platelets), homocysteine, plasma glucose and serum insulin. A detailed description of laboratory measurement procedures is available elsewhere. ${ }^{8}$

\section{Covariates}

Based on the questionnaire design and the frequency distribution of each variable, relevant covariates were categorised as follows:

Race/ethnicity: non-Hispanic white (white), non-Hispanic black (black), Mexican American and other.

Educational attainment: less than high school, high school (including general equivalency diploma) and more than high school.

Annual family income: low (<US\$25000), medium ( $\geq$ US $\$ 25000$ to $<$ US\$55000) and high ( $\geq$ US\$55000).

Leisure time physical activity (over the past 30 days): inactive ( $<10 \mathrm{~min}$ of moderate activities), moderate ( $\geq 10 \mathrm{~min}$ of moderate and $<10 \mathrm{~min}$ of vigorous activities) and active ( $\geq 10 \mathrm{~min}$ of vigorous activities). Vigorous activities mean any physical activities in leisure time that cause 'heavy sweating or large increases in breathing or heart rate'. Moderate activities mean any physical activities in leisure time that cause 'only light sweating or a slight to moderate increase in breathing or heart rate'.

Cigarette smoking status: current smokers (had smoked $\geq 100$ cigarettes in lifetime and still smoked at the time of interview), former smokers (had smoked $\geq 100$ cigarettes in lifetime but did not smoke at the time of interview) and never smokers (had not smoked $\geq 100$ cigarettes in lifetime). For current and former smokers, pack-years of cigarette smoking were calculated and divided into quintiles.

Passive smoking was defined by self-report of second-hand smoke at home or in the workplace. 
Alcohol drinking: participants who had at least 12 drinks of alcoholic beverage in 1 year were defined as alcohol drinkers (a drink means a 12-ounce can of beer, a 4-ounce glass of wine or an ounce of spirits).

Hearing loss: defined as self-report of 'a little trouble hearing', 'a lot of trouble hearing' or 'deafness'; otherwise it was defined as not self-reporting hearing loss. ${ }^{13}$

\section{Statistical analysis}

The characteristics of the participants in the different exposure groups were compared using a Wald $\chi^{2}$ test for categorical variables and t test or analysis of variance for continuous variables. To determine the associations between occupational noise (predictor variable) and the odds of cardiovascular diseases (dependent variable), we performed bivariable (model 1) and multivariable (model 2 and model 3 ) logistic regression analyses using the never exposed group as the reference category. In the multivariable analysis, we adjusted for age, sex, race/ethnicity, BMI (quintiles), educational attainment, leisure time physical activity, cigarette smoking and diabetes as the main analysis (model 2). Furthermore, to control for the influence of annual family income, pack-years of cigarette smoking (quintiles), passive smoking, alcohol drinking, waist circumference (quin- tiles) and total cholesterol level (quintiles), based on model 2 we additionally adjusted for these covariates in model 3 .

We examined the linearity assumption between accumulated exposure time and log odds ratio of $\mathrm{CHD}$ by categorising accumulated exposure time (years) into quartiles and plotting the midpoint of each quartile against the corresponding estimated $\beta$ coefficients from logistic regression. Since the linearity assumption cannot be satisfied, accumulated exposure time was used as categorical variable in the data analysis. ${ }^{14}$

All analyses were performed using SAS Survey Procedures (SAS 9.2, SAS Institute Inc, Cary, North Carolina, USA) to account for the complex sampling design of NHANES. Six-year examination sampling weights were calculated and incorporated into all analyses (unless otherwise specified) to account for differential probabilities of selection, non-coverage and nonresponse of the survey. ${ }^{9}$ Sampling variance was estimated using the Taylor series linearisation.

\section{RESULTS}

In total, 6307 participants were included in the main analysis, of whom $21.2 \%$ were currently exposed to occupational noise. The majority (83.3\%) of these exposed workers were male and their average age was 40 years (range 20-85 years). The median of

Table 1 Characteristics of study participants by noise exposure status (unweighted sample size and weighted percentage)*

\begin{tabular}{|c|c|c|c|c|c|}
\hline & \multirow[b]{2}{*}{$\begin{array}{l}\text { Never exposed } \\
(n=5071)\end{array}$} & \multirow[b]{2}{*}{$\begin{array}{l}\text { Current exposed } \\
(n=1236)\end{array}$} & \multicolumn{3}{|c|}{ Tertiles of accumulated exposure time $\dagger$} \\
\hline & & & $\begin{array}{l}\text { Short } \\
(\mathrm{n}=421)\end{array}$ & $\begin{array}{l}\text { Medium } \\
(\mathrm{n}=399)\end{array}$ & $\begin{array}{l}\text { Long } \\
(n=416)\end{array}$ \\
\hline Age (years), mean (SE) $\ddagger \S$ & $41.0(0.3)$ & $40.0(0.4)$ & $35.9(0.7)$ & $39.6(0.6)$ & $44.4(0.5)$ \\
\hline Male, $\mathrm{n}(\%) \neq \S$ & $2147(41.6)$ & $993(83.3)$ & $331(80.6)$ & $318(84.5)$ & $344(84.9)$ \\
\hline \multicolumn{6}{|l|}{ Ethnicity, n (\%)‡ § } \\
\hline Non-Hispanic white & $2351(68.7)$ & $635(75.5)$ & $204(71.3)$ & $215(76.9)$ & $216(78.3)$ \\
\hline Non-Hispanic black & $1100(12.0)$ & $190(7.5)$ & $73(9.1)$ & $51(6.1)$ & $66(7.4)$ \\
\hline Mexican American & $1155(8.0)$ & $334(9.4)$ & $118(11.1)$ & $102(8.7)$ & $114(8.3)$ \\
\hline \multicolumn{6}{|l|}{ Education, $\mathrm{n}(\%) \neq \S$} \\
\hline$<$ High school & $1208(14.7)$ & $366(19.0)$ & $125(20.6)$ & $111(17.3)$ & $130(19.1)$ \\
\hline High school & $1097(22.3)$ & $391(35.7)$ & $111(28.2)$ & $136(37.6)$ & $144(41.3)$ \\
\hline$>$ High school & $2766(63.0)$ & $479(45.3)$ & $185(51.2)$ & $152(45.1)$ & $142(39.6)$ \\
\hline \multicolumn{6}{|l|}{ Annual family income, $\mathrm{n}(\%) \neq \S$} \\
\hline Low (<US\$25000) & $1444(24.5)$ & $349(21.8)$ & $151(30.5)$ & $112(20.2)$ & $86(14.5)$ \\
\hline $\begin{array}{l}\text { Medium ( } \geq \text { US } \$ 25000 \text { to }<\text { US } \\
\$ 55000 \text { ) }\end{array}$ & $1507(31.4)$ & $442(39.3)$ & $149(39.7)$ & $148(39.9)$ & $145(38.3)$ \\
\hline High ( $\geq$ US $\$ 55000$ ) & $1704(44.1)$ & $360(38.8)$ & $94(29.9)$ & $120(39.8)$ & $146(47.2)$ \\
\hline \multicolumn{6}{|l|}{ Physical activity, n (\%) } \\
\hline Active & $1750(39.4)$ & $442(39.8)$ & $170(44.0)$ & $139(40.3)$ & $133(35.0)$ \\
\hline Moderate & $1322(28.3)$ & $319(27.7)$ & $101(25.8)$ & $109(28.1)$ & $109(29.3)$ \\
\hline Inactive & $1999(32.4)$ & $475(32.5)$ & $150(30.2)$ & $151(31.6)$ & $174(35.8)$ \\
\hline \multicolumn{6}{|l|}{ Cigarette smoking, $\mathrm{n}(\%) \neq \S$} \\
\hline Current smoker & $1087(22.7)$ & $408(35.7)$ & $155(40.3)$ & $134(33.9)$ & $119(32.8)$ \\
\hline Former smoker & $1068(21.6)$ & $302(24.3)$ & $84(19.5)$ & $91(23.8)$ & $127(29.9)$ \\
\hline Never smoker & $2916(55.7)$ & $526(40.0)$ & $182(40.2)$ & $174(42.4)$ & $170(37.3)$ \\
\hline Pack-years, median (IQR) $\ddagger \S$ ฯ & $8.9(2.5-23.8)$ & $11.9(3.9-24.8)$ & $10.6(3.5-21.8)$ & $11.8(3.9-24.7)$ & $13.1(4.9-27.4)$ \\
\hline Passive smoking, $\mathrm{n}(\%) \neq \S$ & $1444(28.9)$ & $627(53.4)$ & $230(56.3)$ & $198(51.0)$ & $199(52.8)$ \\
\hline Alcohol drinking, $\mathrm{n}(\%) \neq \S$ & $3322(74.3)$ & $948(84.7)$ & $326(86.1)$ & $310(84.2)$ & $312(83.8)$ \\
\hline BMI $\left(\mathrm{kg} / \mathrm{m}^{2}\right)$, mean (SE)§ & $27.9(0.1)$ & $28.1(0.2)$ & $27.7(0.3)$ & $27.9(0.3)$ & $28.8(0.3)$ \\
\hline Waist circumference $(\mathrm{cm})$, mean $(\mathrm{SE}) \neq \S$ & $94.3(0.3)$ & $97.9(0.5)$ & $96.7(0.8)$ & $97.1(0.8)$ & $99.8(0.7)$ \\
\hline Pulse rate $(\mathrm{bpm})$, mean $(\mathrm{SE}) \neq \S$ & $72.5(0.3)$ & $70.1(0.5)$ & $71.3(0.7)$ & $69.6(0.8)$ & $69.5(0.7)$ \\
\hline $\operatorname{SBP}(\mathrm{mm} \mathrm{Hg})$, mean $(\mathrm{SE}) \neq \S$ & $119.5(0.4)$ & $121.3(0.5)$ & $120.0(0.9)$ & $121.0(1.0)$ & $123.0(0.8)$ \\
\hline $\mathrm{DBP}(\mathrm{mm} \mathrm{Hg})$, mean $(\mathrm{SE}) \neq \S$ & $72.6(0.3)$ & $74.2(0.4)$ & $72.5(0.6)$ & $74.3(0.8)$ & $76.0(0.6)$ \\
\hline Hearing loss, $\mathrm{n}(\%) \neq \S$ & $783(16.1)$ & $349(31.6)$ & $93(25.5)$ & $108(31.2)$ & $148(38.1)$ \\
\hline
\end{tabular}

\footnotetext{
BMI, body mass index; DBP, diastolic blood pressure; IQR, interquartile range; SBP, systolic blood pressure; SE, standard error.

${ }^{*}$ Sample size is unweighted, percentage in parentheses is weighted to account for multistage probability sampling design.

†Tertiles of accumulated exposure time: short $0-0.3$ years, medium $0.4-1.5$ years, long $1.6-18.8$ years.

$\neq \mathrm{p}<0.05$ for the comparison between the current exposed group and the never exposed group.

$\S p<0.05$ for the comparison between tertile groups and the never exposed group.

$\uparrow$ Median (interquartile range) for current and former cigarette smokers.
} 
accumulated exposure time was 8.8 months (interquartile range 2.4-23.6 months)

Compared to those in the never exposed group, participants in the current exposed group were more likely to be white, have a lower educational level, be a current cigarette smoker, be exposed to second-hand smoke and drink alcoholic beverages. On average, the exposed participants had higher BMI and larger waist circumference; current or former smokers tended to smoke more cigarettes (table 1).

Strikingly, participants in the long-term exposed group had a two- to threefold increase in odds ratios (OR) for angina pectoris, $\mathrm{MI}$ and $\mathrm{CHD}$ (table 2). After adjustment for various covariates in model 2 and model 3 , the observed associations for angina pectoris and $\mathrm{CHD}$ remained statistically significant, with the corresponding ORs being 2.91 (95\% CI 1.35 to 6.26) and 2.04 (95\% CI 1.16 to 3.58 ), respectively (table 2 ). The relevant results based on the longest job are presented in etable 1 in the online appendix.

Figure 1 shows adjusted log ORs and 95\% CIs for CHD in the current exposed group compared with the never exposed group after stratification by relevant characteristics and adjustment for all other covariates in model 2. The association between noise exposure and CHD was stronger for participants aged less than 50 years, men and current smokers. When the main analysis was restricted to participants aged less than 50 years $(n=4543)$ and adjusted for the covariates in model 2 , ORs (95\% CIs) for angina pectoris, MI and CHD were 3.66 (1.85 to 7.24), 4.27 (1.50 to 12.1) and 4.31 (2.26 to 8.19), respectively, for the current exposed group compared with the never exposed group.
Overall, participants exposed to occupational noise tended to have elevated systolic and/or diastolic blood pressure and decreased pulse rate in bivariable analysis, but the associations were not significant after adjustment for covariates in model 2 or model 3. There was no significant increase in self-reported hypertension and general hypertension (table 3). For hypertension subtypes, long-term exposure to occupational noise was associated with increased odds of IDH in bivariable (OR 2.92, 95\% CI 1.71 to 4.98 ) and fully adjusted multivariable analysis (OR 2.23, $95 \%$ CI 1.21 to 4.12 ). There were no significant associations between noise exposure and other hypertension subtypes (table 3).

In bivariable analysis, participants in the long-term exposure group had a significant increase in levels of total cholesterol, LDLcholesterol, triglyceride, homocysteine, glucose and haematocrit and a significant decrease in the level of HDL-cholesterol. After adjustment for covariates in model 2 and model 3 , there were no significant differences in these measurements compared to participants in the never exposed group (table 4).

To examine the potential influence of missing data, we performed bivariable analyses to compare effect estimates for $\mathrm{CHD}$ (current exposed group and tertile groups compared to never exposed group) between participants with complete noise exposure data $(n=6835)$, with complete covariate data in model $2(n=6307)$ and with complete covariate data in model 3 $(n=4829)$. The unadjusted effect estimates from the three groups were proximate, suggesting that excluding participants with missing covariate data would not substantially affect the results of this study.

Table 2 Odds ratios ( $95 \%$ Cls) of self-reported cardiovascular diseases by tertiles of accumulated noise exposure time*

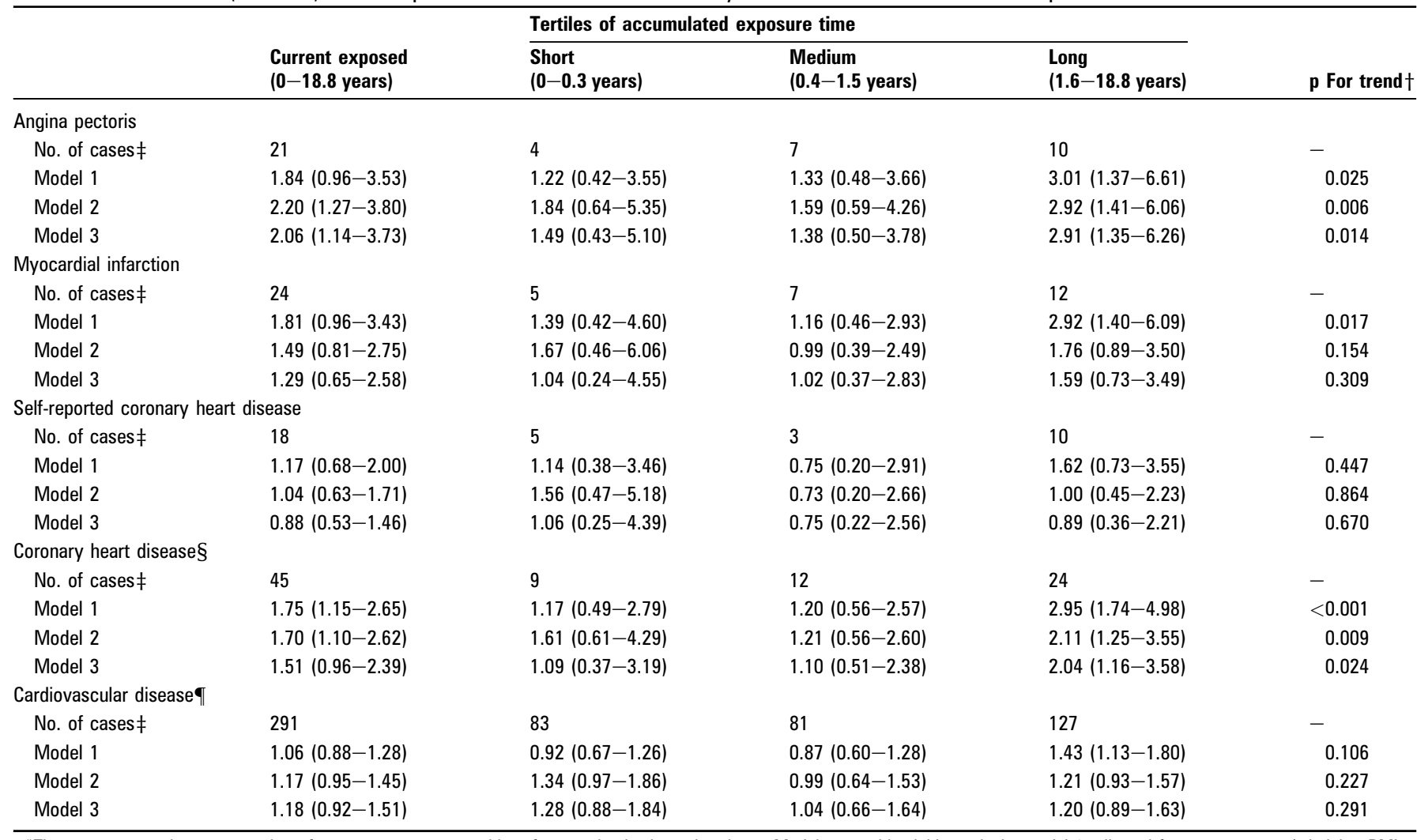

*The never exposed group was the reference category; see table 1 for sample size in each column. Model 1 was bivariable analysis; model 2 adjusted for age, sex, race/ethnicity, BMI, educational level, physical activity, cigarette smoking and diabetes; model 3 additionally adjusted for annual family income, pack-years of cigarette smoking, passive smoking in the workplace or at home, alcohol drinking, waist circumference and total cholesterol.

tp Is for linear trend across tertile groups.

‡For model 1 and model 2. The number of cases in model 3 is smaller due to missing data.

SIncludes self-reported angina pectoris, myocardial infarction and coronary heart disease.

IIncludes coronary heart disease, self-reported hypertension, stroke and congestive heart failure. 


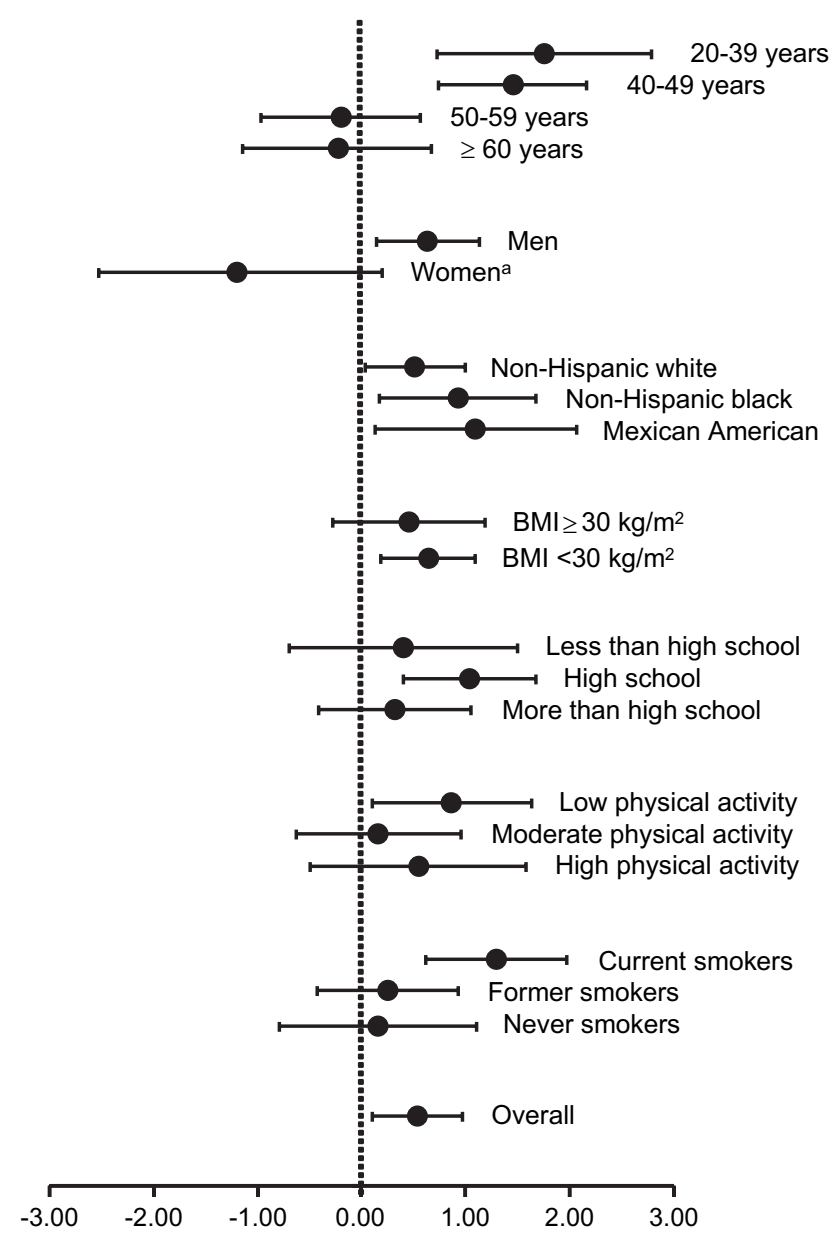

Adjusted log odds ratio $(95 \% \mathrm{Cl})$ for coronary heart disease

Figure 1 Adjusted log odds ratios (95\% Cls) of coronary heart disease for the current exposed group compared with the never exposed group, stratified by selected characteristics and adjusted for all other covariates in model 2. ${ }^{\mathrm{a}} 243$ women (three CHD cases) in the current exposed group, 2924 women (56 CHD cases) in the never exposed group.

\section{DISCUSSION}

This study was based on a large nationally representative sample with extensive individual information, which allowed for better control of known cardiovascular risk factors. We found that long-term exposure to self-reported loud noise in the workplace was associated with a nearly threefold increase in the prevalence of angina pectoris and a twofold increase in the prevalence of $\mathrm{CHD}$ and $\mathrm{IDH}$, respectively. The association with angina pectoris, MI or CHD was particularly strong for participants aged less than 50 years, men and current smokers. We did not find a substantial elevation in the levels of blood lipids and circulating inflammatory mediators.

There have been several previous studies that have examined the associations between exposure to occupational noise and coronary events, but the results have not been fully consistent. A 24-year retrospective cohort study of Canadian sawmill workers found that exposure to occupational noise was associated with a two- to fourfold increase in the risk of acute MI mortality. ${ }^{6}$ Similarly, a 13-year prospective cohort study of Finnish male workers found a 1.5 -fold increase in CHD hospitalisation or mortality for those exposed to occupational noise. ${ }^{15}$ In a case-control study in Berlin, self-reported exposure to loud occupational noise was associated with a 1.4-3.8-fold increase in acute MI hospitalisation. ${ }^{16}$ However, a matched case-control study of male nuclear power workers in England did not find a robust association between noise exposure and $\mathrm{CHD}$ mortality. ${ }^{7}$ The reasons for the discrepancies may be related to differences in workplace noise level, duration of exposure, exposure assessment method, study design and sample size across different studies. Notably, in the present study when we used the longest job (either former or current job) rather than current job to evaluate noise exposure and repeat the analyses, we did not find a robust association with CHD (see etable 1 in the online appendix), suggesting that the morbidity of CHD was dependent on noise stimulation and might decrease after termination of the exposure. This phenomenon has also been observed in previous studies ${ }^{6} 15$ and may partly explain the null association in the case-control study in which $80 \%$ of $\mathrm{CHD}$ deaths occurred after leaving their noisy jobs. ${ }^{7}$

The findings from previous studies on associations between occupational noise and blood pressure or hypertension are not consistent. ${ }^{4}$ A meta-analysis of cross-sectional studies indicated that exposure to occupational noise was associated with an increase in systolic blood pressure or the prevalence of hypertension; no significant association was found with diastolic blood pressure. ${ }^{4}$ Nevertheless, there was substantial heterogeneity in effect estimates across original studies. ${ }^{4}$ No previous studies have examined the relationships between noise exposure and hypertension subtypes. In the present study, we did not find significant alterations in the levels of SBP, DBP and pulse rate and the prevalence of self-reported hypertension or general hypertension ( $\mathrm{SBP} \geq 140 \mathrm{~mm} \mathrm{Hg}, \mathrm{DBP} \geq 90 \mathrm{~mm} \mathrm{Hg}$, or selfreported hypertension) for exposed participants. But we found that noise exposure was associated with increased prevalence of $\mathrm{IDH}$ in an exposure-response fashion. Our findings are largely consistent with those from a controlled exposure study, which showed that there was an increase in DBP but no change in SBP and heart rate for 18 healthy human volunteers exposed to occupational noise of $95 \mathrm{dBA}$ for $20 \mathrm{~min}^{17}$

IDH develops due to increased arteriolar resistance without evident large artery atherosclerosis, and is more common in young and middle-aged people. ${ }^{18} 19 \mathrm{IDH}$ is an independent predictor of coronary events ${ }^{2021}$; a large cohort study showed that compared to normotensive participants, those with IDH at baseline had a $55 \%$ and a $47 \%$ increase in CHD incidence and mortality, respectively, during an 8-year follow-up period. ${ }^{21}$ Accordingly, the increased prevalence of IDH may be a pathway for the association between noise exposure and CHD in the present study.

A potential mechanism linking noise exposure to myocardial ischaemia or infarction is that loud occupational noise may serve as a potent external stressor, similar to sudden emotional stress ${ }^{22}$ and physical exertion, ${ }^{23}$ to activate the sympathetic nervous system and endocrine system, ${ }^{24}$ leading to coronary vasoconstriction (spasm) and subsequent partial or complete coronary occlusion $^{25-27}$ in participants with or without pre-existing coronary atherosclerotic plaque 28 or leading to physical disruption of vulnerable plaque and subsequent thrombosis. ${ }^{29} 30$ We did not find substantial increases in the levels of important cardiovascular risk factors such as blood lipids ${ }^{31}$ and circulating inflammatory mediators. ${ }^{32}$ These cardiovascular risk factors may not play important roles for the observed associations in this relatively young working population.

This study has several limitations that should be considered. First, the study was based on currently employed workers. Because of the healthy worker survivor effect in working populations, ${ }^{33}$ this study is prone to selection bias, especially for the older workers. Figure 1 shows that noise exposure was not 
Table 3 Mean differences (95\% Cls) in pulse rate and blood pressure, and odds ratios (95\% Cls) of hypertension subtypes, by tertiles of accumulated noise exposure time*

\begin{tabular}{|c|c|c|c|c|c|}
\hline & \multirow[b]{2}{*}{$\begin{array}{l}\text { Current exposed } \\
\text { (0-18.8 years) }\end{array}$} & \multicolumn{3}{|c|}{ Tertiles of accumulated exposure time } & \multirow[b]{2}{*}{ p For trend $\dagger$} \\
\hline & & $\begin{array}{l}\text { Short } \\
\text { (0-0.3 years) }\end{array}$ & $\begin{array}{l}\text { Medium } \\
(0.4-1.5 \text { years })\end{array}$ & $\begin{array}{l}\text { Long } \\
(1.6-18.8 \text { years })\end{array}$ & \\
\hline \multicolumn{6}{|c|}{ Pulse rate $(\mathrm{bpm}) \neq$} \\
\hline Model 2 & $-1.29(-2.50$ to -0.08$)$ & $-0.59(-2.38$ to 1.21$)$ & $-1.81(-3.49$ to -0.13$)$ & $-1.49(-3.06$ to 0.08$)$ & 0.019 \\
\hline Model 3 & $-1.27(-2.62$ to 0.09$)$ & $-0.83(-2.78$ to 1.13$)$ & $-1.59(-3.36$ to 0.18$)$ & $-1.38(-3.47$ to 0.72$)$ & 0.038 \\
\hline \multicolumn{6}{|c|}{ Systolic blood pressure $(\mathrm{mm} \mathrm{Hg}) \ddagger$} \\
\hline Model 3 & $0.39(-0.90$ to 1.67$)$ & $1.56(-0.16$ to 3.28$)$ & $0.11(-2.03$ to 2.24$)$ & $-0.54(-2.44$ to 1.37$)$ & 0.850 \\
\hline \multicolumn{6}{|c|}{ Diastolic blood pressure $(\mathrm{mm} \mathrm{Hg}) \neq$} \\
\hline Model 1 & 1.62 (0.72 to 2.53$)$ & $-0.13(-1.37$ to 1.11$)$ & 1.69 (0.02 to 3.36$)$ & 3.33 (1.97 to 4.69$)$ & $<0.001$ \\
\hline Model 2 & $0.58(-0.29$ to 1.46$)$ & $-0.08(-1.24$ to 1.07$)$ & $0.71(-0.78$ to 2.21$)$ & $1.14(-0.20$ to 2.49$)$ & 0.077 \\
\hline Model 3 & $0.74(-0.40$ to 1.88$)$ & $0.03(-1.54$ to 1.59$)$ & $1.22(-0.50$ to 2.95$)$ & $0.97(-0.49$ to 2.44$)$ & 0.102 \\
\hline \multicolumn{6}{|c|}{ Isolated systolic hypertension§ } \\
\hline Model 1 & 2.03 (1.33 to 3.09$)$ & $1.58(0.94$ to 2.66$)$ & $1.64(0.88$ to 3.04$)$ & 2.92 (1.71 to 4.98$)$ & $<0.001$ \\
\hline Model 2 & $1.43(0.90$ to 2.25$)$ & $1.22(0.70$ to 2.13$)$ & 1.15 (0.58 to 2.26$)$ & 1.90 (1.07 to 3.37$)$ & 0.058 \\
\hline Model 3 & 1.52 (0.92 to 2.53$)$ & $1.12(0.60$ to 2.10$)$ & 1.26 (0.58 to 2.74$)$ & 2.23 (1.21 to 4.12$)$ & 0.025 \\
\hline \multicolumn{6}{|c|}{ Systodiastolic hypertension§ } \\
\hline Model 1 & $1.02(0.70$ to 1.50$)$ & 1.31 (0.76 to 2.27$)$ & $1.12(0.60$ to 2.07$)$ & 0.64 (0.31 to 1.34$)$ & 0.584 \\
\hline Model 2 & $0.96(0.77$ to 1.19$)$ & $1.73(0.97$ to 3.05$)$ & $1.26(0.64$ to 2.49$)$ & $0.54(0.25$ to 1.17$)$ & 0.473 \\
\hline Model 3 & $1.00(0.61$ to 1.64$)$ & $1.80(0.94$ to 3.42$)$ & 1.06 (0.48 to 2.38$)$ & $0.45(0.19$ to 1.08$)$ & 0.237 \\
\hline \multicolumn{6}{|c|}{ Self-reported hypertension§ } \\
\hline Model 1 & 0.97 (0.78 to 1.21$)$ & 0.84 (0.60 to 1.17$)$ & 0.88 (0.59 to 1.31$)$ & $1.22(0.93$ to 1.61$)$ & 0.651 \\
\hline Model 2 & $1.10(0.87$ to 1.39$)$ & $1.22(0.89$ to 1.69$)$ & 1.03 (0.66 to 1.62$)$ & $1.07(0.78$ to 1.46$)$ & 0.611 \\
\hline Model 3 & $1.12(0.86$ to 1.47$)$ & $1.21(0.85$ to 1.72$)$ & $1.10(0.69$ to 1.75$)$ & $1.08(0.76$ to 1.54$)$ & 0.562 \\
\hline
\end{tabular}

*The never exposed group was the reference category, please see table 1 for sample size in each column. Model 1 was bivariable analysis; model 2 adjusted for age, sex, ethnicity, BMl, educational level, physical activity, cigarette smoking and diabetes; model 3 additionally adjusted for annual family income, pack-years of cigarette smoking, passive smoking in the workplace or at home, alcohol drinking, waist circumference and total cholesterol.

$\mathrm{tp}$ Is for linear trend across tertile groups.

$\neq \beta$ Coefficient $(95 \% \mathrm{Cl})$ from linear regression represents mean difference between a specific exposed group and the never exposed group.

§Odds ratio $(95 \% \mathrm{Cl})$.

ॠSystolic blood pressure $\geq 140 \mathrm{~mm} \mathrm{Hg}$, diastolic blood pressure $\geq 90 \mathrm{~mm} \mathrm{Hg}$, or self-reported hypertension.

associated with CHD for older participants. This observation may be true because the risk of hearing loss such as noise-induced hearing loss, substantially increases with age and years spent in noisy workplaces. ${ }^{13} 3435$ As a result, older workers are more likely to have hearing loss and thus are not sensitive to some types of occupational noise. However, it is also possible that this null association may partly result from selection bias. After a long period of employment selection, those older workers vulnerable to workplace noise or with cardiovascular diseases might have been more likely to move away from their original noisy jobs or some might have died from CHD while having noisy jobs. This cross-sectional survey thus cannot capture these workers, leading to false null associations. Similarly, because of the selection bias, although the effect estimates in our study are striking, the true adverse cardiovascular effects associated with occupational noise may be even larger for this population. Based on the present crosssectional study of currently employed workers, null associations should be interpreted cautiously. Future cohort studies are needed to clarify these observed associations.

Second, noise exposure was based on self-assessment of exposure to loud occupational noise. Because of differences in subjective judgement, misclassification of noise exposure might have occurred, which might bias the effect estimates towards the null. Nevertheless, table 1 shows that both self-reported noise exposure and accumulated exposure time strongly predict the prevalence of hearing loss in bivariable and multivariable models $(p<0.001)$, indicating that self-reported noise exposure was able to reflect actual noise exposure in the workplace, although in the present study hearing loss was also based on self-reports and thus prone to recall bias. In addition, previous studies have shown that self-report is a valid approach in exposure assessment of occupational noise. ${ }^{36} 37$ In general, selfreported loud occupational noise approximately corresponds to actual workplace noise levels of $80-85 \mathrm{dBA}$ and over. ${ }^{36} 37$

Third, cardiovascular disease was defined as self-report of physician diagnosis, which might have caused disease misclassification due to incorrect recall or lack of knowledge about disease classification. For example, in this study, $50 \%$ of participants with self-reported angina pectoris and $43 \%$ of participants with selfreported MI did not report $\mathrm{CHD}$. This misclassification may partly explain the null association between noise exposure and self-reported CHD (table 2). However, given the severity of 
Table 4 Serum biochemistry profiles by noise exposure status

\begin{tabular}{|c|c|c|c|c|c|}
\hline & \multirow[b]{2}{*}{$\begin{array}{l}\text { Never exposed } \\
(n=5071)\end{array}$} & \multirow[b]{2}{*}{$\begin{array}{l}\text { Current exposed } \\
(n=1236)\end{array}$} & \multicolumn{3}{|c|}{ Tertiles of accumulated exposure time } \\
\hline & & & $\begin{array}{l}\text { Short } \\
(n=421)\end{array}$ & $\begin{array}{l}\text { Medium } \\
(n=399)\end{array}$ & $\begin{array}{l}\text { Long } \\
(n=416)\end{array}$ \\
\hline Leukocytes $\left(\times 10^{9} / \mathrm{l}\right)$, mean (SE) & $7.2(0.1)$ & $7.4(0.1)^{*}$ & $7.6(0.1)^{*} \dagger$ & $7.2(0.1)$ & $7.3(0.1)$ \\
\hline Platelets $\left(\times 10^{9} / \mathrm{I}\right)$, mean $(\mathrm{SE})$ & $270.0(1.3)$ & $265.8(1.7)^{*} \dagger$ & $266.7(3.6)$ & $266.6(3.3)$ & $263.9(3.3)$ \\
\hline Haematocrit (\%), mean (SE) & $42.4(0.1)$ & $45.0(0.2)^{*}$ & $45.0(0.3)^{*}$ & $45.0(0.3)^{*}$ & $45.1(0.3)^{*}$ \\
\hline Fibrinogen $(g / l)$, mean $(S E) \ddagger$ & $3.52(0.03)$ & $3.47(0.06)$ & $3.47(0.07)$ & $3.40(0.08)$ & $3.52(0.06)$ \\
\hline $\mathrm{CRP}(\mathrm{mg} / \mathrm{l})$, median (IOR) & $0.18(0.06-0.42)$ & $0.15(0.07-0.33)^{*}$ & $0.13(0.06-0.33)^{*}$ & $0.14(0.06-0.31)^{*}$ & $0.17(0.08-0.36)$ \\
\hline Homocysteine $(\mu \mathrm{mol} / \mathrm{l})$, mean (SE) & $8.1(0.1)$ & $8.7(0.1)^{*}$ & $8.3(0.1)$ & $9.0(0.3)^{*}$ & $8.9(0.1)^{*}$ \\
\hline Total cholesterol $(\mathrm{mmol} / \mathrm{l})$, mean (SE) & $5.19(0.02)$ & $5.25(0.05)$ & $5.08(0.10)$ & $5.17(0.07)$ & $5.50(0.08)^{*}$ \\
\hline HDL-cholesterol (mmol/l), mean (SE) & $1.38(0.01)$ & $1.24(0.01)^{*}$ & $1.24(0.02)^{*}$ & $1.23(0.02)^{*}$ & $1.25(0.02)^{*}$ \\
\hline LDL-cholesterol $(\mathrm{mmol} / \mathrm{l})$, mean $(\mathrm{SE}) \S$ & $3.10(0.03)$ & $3.15(0.04)$ & $2.99(0.09)$ & $3.12(0.07)$ & $3.31(0.07)^{*}$ \\
\hline Triglyceride $(\mathrm{mmol} / \mathrm{l}) \S$ q & $1.28(1.02)$ & $1.40(1.03)$ & $1.25(1.05)$ & $1.32(1.06)$ & $1.61(1.05)^{*}$ \\
\hline Plasma glucose $(\mathrm{mmol} / \mathrm{l}) \S$ ๆ & $5.34(1.01)$ & $5.49(1.01)^{*}$ & $5.33(1.01)$ & $5.41(1.01)$ & $5.72(1.01)^{*}$ \\
\hline Serum insulin $(\mathrm{pmol} / \mathrm{l}) \S \boldsymbol{\Phi}^{* *}$ & $42.3(1.0)$ & $42.0(1.1)$ & $42.5(1.1)$ & $40.5(1.1)$ & $43.2(1.1)$ \\
\hline
\end{tabular}

CRP, C-reactive protein; HDL, high-density lipoprotein cholesterol; IOR, interquartile range; LDL, low-density lipoprotein cholesterol; SE, standard error.

${ }^{*} \mathrm{p}<0.05$ compared with the never exposed group in bivariable analysis.

tp $<0.05$ compared with the never exposed group after adjustment for age, sex, ethnicity, BMI, educational level, leisure time physical activity, cigarette smoking and diabetes (model 2)

$\neq$ Only available during NHANES 1999-2000, 2001-2002 for participants aged 40 years and older. Four-year weights were incorporated into this analysis. Number of participants in the current/never exposed group: 422/1721.

§This is a morning fasting sample; 6 -year fasting weights were incorporated into the analysis. Number of participants in the current/never exposed group: LDL-cholesterol 519/2147, triglyceride $535 / 2202$, plasma glucose $537 / 2229$, serum insulin 533/2197.

$\uparrow$ Geometric mean (SE of the geometric mean).

**Serum insulin level was measured using a Pharmacia method during NHANES 1999-2002 but a Tosoh method during NHANES 2003-2004. The Pharmacia values were transferred into the

Tosoh values to keep insulin values consistent; two participants with negative transferred values were excluded from the analysis.

cardiovascular diseases, participants who had a specific CHD such as angina pectoris or MI were less likely to mistakenly report the specific disease. Therefore, the results based on self-reported angina pectoris, MI and all types of CHD should be reliable.

Finally, this study did not take into account other occupational or environmental factors that are associated with cardiovascular diseases such as shift work and physical workload, ${ }^{15}$ noise exposure from road traffic, ${ }^{38}$ fine particulate air pollution in the workplace and residence. ${ }^{39}$ These relevant factors might potentially affect the effect estimates in the present study. However, there is no evidence that these factors are associated with exposure to occupational noise. These relevant factors may be non-differentially distributed in the exposed group and the never exposed group and thus may not substantially affect the observed associations.

The present study did not include participants previously but not currently exposed to occupational noise (currently employed or unemployed workers) since the temporal relationship between noise exposure and the development of CHD is uncertain (the relevant analysis is presented in etables 1 and 2 in the online appendix). On the one hand, it is possible that CHD might occur when participants worked in the noisy jobs but moved away from the jobs due to the disease. Obviously, this situation suggests that previous noise exposure was associated with the disease. On the other hand, it is also possible that some workers might move away from their noisy jobs without having the disease and the disease might occur later or even after retirement without the stimulation of occupational noise. In this situation, previous noise exposure (as an external physical stressor) was less likely to be associated with the disease. This cross-sectional study cannot distinguish these two different circumstances, and therefore cannot determine whether previous exposure to occupational noise was associated with CHD. Future prospective cohort studies are necessary to clarify the relationship between previous noise exposure and the risk of $\mathrm{CHD}$.

\section{CONCLUSIONS}

Using a large nationally representative sample with extensive individual information, we found that long-term exposure to self-reported loud noise in the workplace was associated with a nearly threefold increase in the prevalence of angina pectoris, and a twofold increase in the prevalence of $\mathrm{CHD}$ and $\mathrm{IDH}$. These associations were particularly strong for young workers under 50 years of age. This study suggests that excess noise exposure in the workplace is an important occupational health issue and deserves special attention.

Funding Wengi Gan was supported by the Canadian Institutes of Health Research Frederick Banting and Charles Best Canada Graduate Scholarship (2008-2011) and by the Michael Smith Foundation for Health Research Senior Graduate Studentship (2008-2011).

\section{Competing interests None.}

Provenance and peer review Not commissioned; externally peer reviewed.

\section{REFERENCES}

1. Nelson DI, Nelson RY, Concha-Barrientos $M$, et al. The global burden of occupational noise-induced hearing loss. Am J Ind Med 2005:48:446-58.

2. Tak S, Davis RR, Calvert GM. Exposure to hazardous workplace noise and use of hearing protection devices among US workers-NHANES, 1999-2004. Am J Ind Med 2009;52:358-71

3. Passchier-Vermeer W, Passchier WF. Noise exposure and public health. Environ Health Perspect 2000;108:123-31

4. van Kempen EE, Kruize $\mathrm{H}$, Boshuizen $\mathrm{HC}$, et al. The association between noise exposure and blood pressure and ischemic heart disease: a meta-analysis. Environ Health Perspect 2002;110:307-17.

5. Jarup L, Babisch W, Houthuijs D, et al. Hypertension and exposure to noise near airports: the HYENA study. Environ Health Perspect 2008;116:329-33.

6. Davies HW, Teschke K, Kennedy SM, et al. Occupational exposure to noise and mortality from acute myocardial infarction. Epidemiology 2005;16:25-32.

7. McNamee R, Burgess G, Dippnall WM, et al. Occupational noise exposure and ischaemic heart disease mortality. Occup Environ Med 2006:63:813-19.

8. National Center for Health Statistics. Centers for Disease Control and Prevention National Health and Nutrition Examination Survey. http://www.cdc.gov/nchs/about/ major/nhanes/datalink.htm (accessed 25 Apr 2010).

9. National Center for Health Statistics, Centers for Disease Control and Prevention The National Health and Nutrition Examination Survey (NHANES) Analytic and Reporting Guidelines. http://www.cdc.gov/nchs/data/nhanes/nhanes_03_04/ nhanes_analytic_guidelines_dec_2005.pdf (accessed 25 Apr 2010).

10. National Center for Health Statistics. Centers for Disease Control and Prevention Response Rates \& CPS Population Totals. http://www.cdc.gov/nchs/nhanes/ nhanes cps totals.htm (accessed 25 Apr 2010).

11. Perloff D, Grim C, Flack J, et al. Human blood pressure determination by sphygmomanometry. Circulation 1993;88:2460-70.

12. Chobanian AV, Bakris GL, Black HR, et al. The Seventh Report of the Joint Nationa Committee on Prevention, Detection, Evaluation and Treatment of High Blood Pressure: the JNC 7 report. JAMA 2003;289:2560-72. 
13. Agrawal Y, Platz EA, Niparko JK. Prevalence of hearing loss and differences by demographic characteristics among US adults: data from the National Health and Nutrition Examination Survey, 1999-2004. Arch Intern Med 2008:168:1522-30.

14. Hosmer DW, Lemeshow S. Applied logistic Regression. 2nd edn. New York, USA: Wiley, 2000

15. Virkkunen H, Harma M, Kauppinen T, et al. The triad of shift work, occupational noise and physical workload and risk of coronary heart disease. Occup Environ Med 2006;63:378-86.

16. Ising $\mathbf{H}$, Babisch W, Kruppa B, et al. Subjective work noise: a major risk factor in myocardial infarction. Soz Praventivmed 1997;42:216-22.

17. Andren L, Hansson L, Bjorkman M, et al. Noise as a contributory factor in the development of elevated arterial pressure. A study of the mechanisms by which noise may raise blood pressure in man. Acta Med Scand 1980;207:493-8.

18. Verdecchia P, Angeli F. Natural history of hypertension subtypes. Circulation 2005;111:1094-6.

19. Fang J, Madhavan $\mathrm{S}$, Cohen $\mathrm{H}$, et al. Isolated diastolic hypertension. A favorable finding among young and middle-aged hypertensive subjects. Hypertension 1995;26:377-82.

20. Hozawa A, Ohkubo T, Nagai K, et al. Prognosis of isolated systolic and isolated diastolic hypertension as assessed by self-measurement of blood pressure at home: the Ohasama study. Arch Intern Med 2000;160:3301-6.

21. Kelly TN, Gu D, Chen J, et al. Hypertension subtype and risk of cardiovascular disease in Chinese adults. Circulation 2008;118:1558-66.

22. Wittstein IS, Thiemann DR, Lima JA, et al. Neurohumoral features of myocardial stunning due to sudden emotional stress. N Engl J Med 2005;352:539-48.

23. Mittleman MA, Maclure M, Tofler GH, et al. Triggering of acute myocardial infarction by heavy physical exertion. Protection against triggering by regular exertion. Determinants of Myocardial Infarction Onset Study Investigators. N Engl J Med 1993;329:1677-83.

24. Remme WJ. The sympathetic nervous system and ischaemic heart disease. Eur Heart J 1998;19:F62-71.

25. Pupita G, Maseri A, Kaski JC, et al. Myocardial ischemia caused by distal coronaryartery constriction in stable angina pectoris. N Engl J Med 1990;323:514-20.
26. Maseri A, Davies G, Hackett D, et al. Coronary artery spasm and vasoconstriction. The case for a distinction. Circulation 1990;81:1983-91.

27. Mohri M, Koyanagi M, Egashira K, et al. Angina pectoris caused by coronary microvascular spasm. Lancet 1998:351:1165-9.

28. Lacy CR, Contrada RJ, Robbins ML, et al. Coronary vasoconstriction induced by mental stress (simulated public speaking). Am J Cardiol 1995:75:503-5.

29. Tofler GH, Muller JE. Triggering of acute cardiovascular disease and potential preventive strategies. Circulation 2006;114:1863-72.

30. Libby $\mathbf{P}$, Theroux P. Pathophysiology of coronary artery disease. Circulation 2005;111:3481-8.

31. Fletcher B, Berra $\mathrm{K}$, Ades $\mathrm{P}$, et al. Managing abnormal blood lipids: a collaborative approach. Circulation 2005;112:3184-209.

32. Libby $\mathbf{P}$, Ridker PM. Inflammation and atherothrombosis - From population biology and bench research to clinical practice. J Am Coll Cardiol 2006:48:A33-A46.

33. Arrighi HM, Hertz-Picciotto I. The evolving concept of the healthy worker survivor effect. Epidemiology 1994:5:189-96.

34. Palmer KT, Griffin MJ, Syddall HE, et al. Occupational exposure to noise and the attributable burden of hearing difficulties in Great Britain. Occup Environ Med 2002:59:634-9.

35. Rabinowitz PM, Galusha D, Dixon-Ernst C, et al. Do ambient noise exposure levels predict hearing loss in a modern industrial cohort? Occup Environ Med 2007:64:53-9.

36. Schlaefer K, Schlehofer B, Schuz J. Validity of self-reported occupational noise exposure. Eur J Epidemiol 2009;24:469-75.

37. Ahmed HO, Dennis JH, Ballal SG. The accuracy of self-reported high noise exposure level and hearing loss in a working population in Eastern Saudi Arabia. Int J Hyg Environ Health 2004:207:227-34.

38. Selander J, Nilsson ME, Bluhm G, et al. Long-term exposure to road traffic noise and myocardial infarction. Epidemiology 2009;20:272-9.

39. Brook RD, Franklin B, Cascio W, et al. Air pollution and cardiovascular disease: a statement for healthcare professionals from the Expert Panel on Population and Prevention Science of the American Heart Association. Circulation 2004:109:2655-71. 